\title{
The double burden of overnutrition and undernutrition in mother-child dyads in Kenya: demographic and health survey data, 2014
}

\author{
Peninah Kinya Masibo ${ }^{1,2}$, Felix Humwa ${ }^{1}$ and Teresia Njoki Macharia ${ }^{1}$ \\ ${ }^{1}$ Global Programs for Research \& Training, Kenya, Affiliate of the University of California San Francisco (UCSF), San Francisco, CA, USA \\ ${ }^{2}$ Department of Nutrition, Moi University, School of Public Health, Nairobi, Kenya
}

(Received 10 June 2019 - Final revision received 26 November 2019 - Accepted 28 November 2019)

Journal of Nutritional Science (2020), vol. 9, e5, page 1 of 12

doi:10.1017/jns.2019.39

Abstract

The double burden of overnutrition and undernutrition is rapidly becoming a public health concern in low- and middle-income countries. We explored the occurrence of mother-child pairs of over- and undernutrition and the contributing factors using the 2014 Kenya Demographic and Health Survey data. A weighted sample of 7830 mother-child pairs was analysed. The children's nutritional status was determined using the WHO 2006 reference standards while maternal nutritional status was determined with BMI. Descriptive statistics, bivariate and multivariate logistic regression analysis were conducted. The proportion of overweight and obese mothers was $26 \%$ (18.8 \% overweight and $7.2 \%$ obese). The prevalence of child stunting, underweight and wasting was $26 \cdot 3,12 \cdot 8$ and $5.1 \%$, respectively. Out of the overweight/obese mothers (weighted $n 2034$ ), $20 \%$ had stunted children, $5.4 \%$ underweight children and $3.1 \%$ wasted children. Overweight/obese mother-stunted child pairs and overweight/obese mother-underweight child pairs were less likely to occur in the rural areas (adjusted OR $(\mathrm{aOR})=0.43 ; P<0 \cdot 01)$ in comparison with those residing in the urban areas $(\mathrm{aOR}=0 \cdot 54 ; P=0 \cdot 01)$. Children aged more than 6 months were more likely to be in the double burden dyads compared with children below 6 months of age $(P<0 \cdot 01)$. The double burden mother-child dyads were more likely to be observed in wealthier households. Mother-child double burden is a notable public health problem in Kenya. Household wealth and urban residence are determinants of the double burden. There is need for target-specific interventions to simultaneously address child undernutrition and maternal overweight/obesity.

Key words: Undernutrition: Overnutrition: Mother-child pairs: Double burden: Kenya: Demographic health survey

A double burden of malnutrition is the coexistence of undernutrition and overnutrition in the same settings either at the individual, household or community level ${ }^{(1)}$. At the individual level, the usual phenotype of the double burden is a stunted child who is also overweight or obese ${ }^{(1)}$. A household-level double burden is the co-existence of at least one member of a household with undernutrition and at least one member with overnutrition while a community-level double burden is when the two ends of the malnutrition spectrum are experienced in the same community, particularly among children ${ }^{(2)}$. The co-existence of over- and undernutrition within the same household is a complex challenge because of the shared household micro-environment. It would be expected that members of the same household have access to analogous foods and that dietary intake at the household level would result in alike nutrition outcomes. The quality of diet at the household level determines the nutritional status outcomes of the household members. Habitual consumption of energydense foods that are poor in nutrients contribute to adult overweight/obesity whilst they deprive young children of essential nutrients that they need for satisfactory growth. In addition, unequal intra-household food distribution may result in the mothers receiving a higher food ration in comparison with their children who receive less ${ }^{(3)}$.

Abbreviations: aOR, adjusted OR; DHS, Demographic and Health Survey; KDHS, Kenya Demographic and Health Survey.

* Corresponding author: Peninah Kinya Masibo, email peninahmasibo@gmail.com and Peninah.Masibo@ucglobalprograms.org 


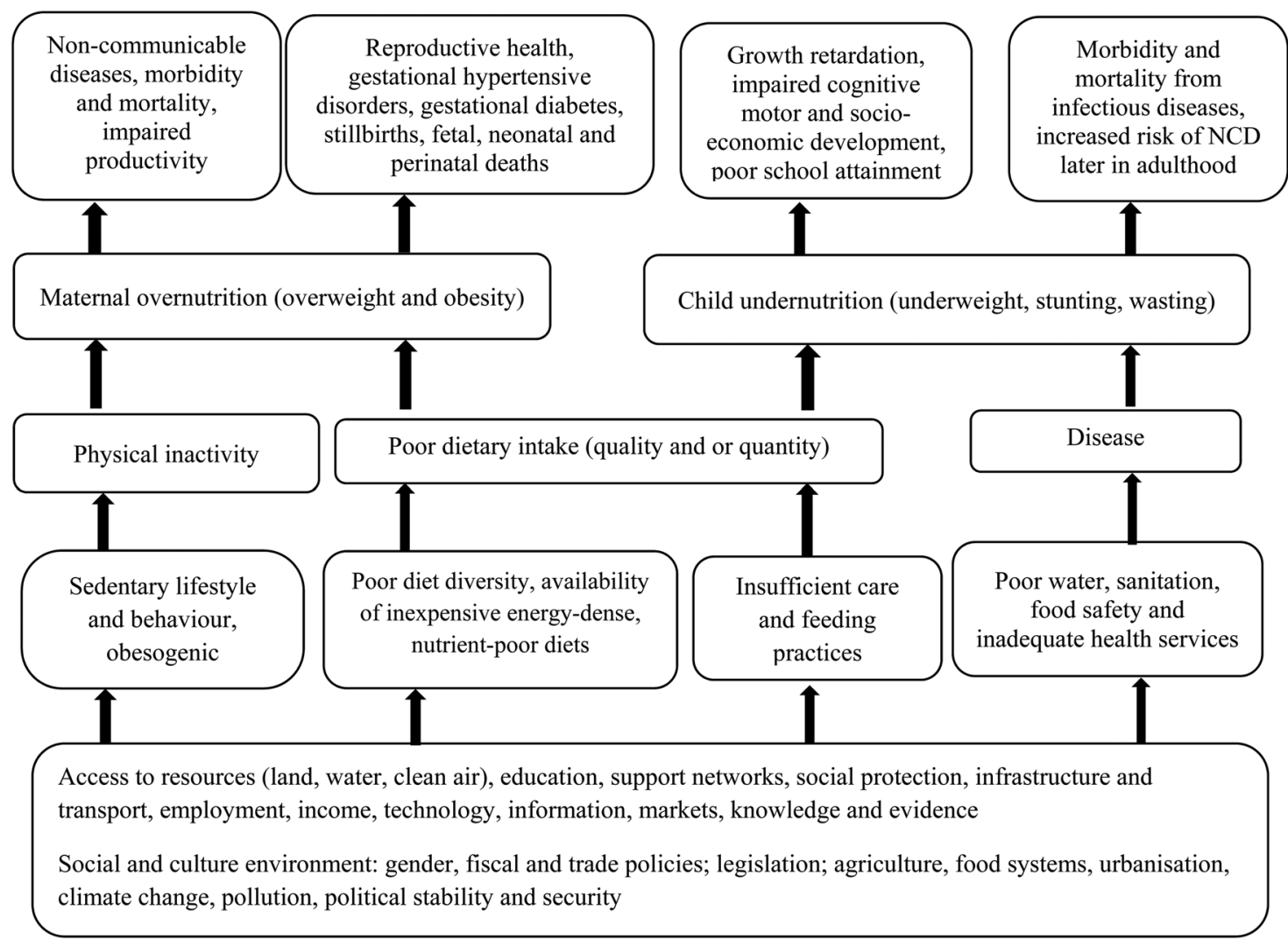

Consequences

\section{Immediate \\ determinants \\ at individual \\ level \\ Underlying \\ causes at \\ household and \\ family levels}

\section{Basic}

determinants at social level

Fig. 1. Conceptual framework for the determinants and outcomes of the dual burden of over- and undernutrition. NCD, non-communicable diseases. Source: adapted from the $\mathrm{WHO}^{(14)}$ and $\mathrm{UNICEF}^{(15)}$.

The double burden phenomenon has been reported in lowand middle-income countries ${ }^{(1,2,4-7)}$. Earlier studies in sub-Saharan Africa demonstrate low double burden rates in households. Garret \& Ruel ${ }^{(8)}$ reported $<10 \%$ prevalence of double burden households in North Africa and sub-Saharan Africa. More recent studies have reported a higher prevalence of double burden mother-child pairs in specific areas of the population. For instance, a study in a large urban informal settlement in Nairobi, Kenya reported up to $40 \%$ prevalence of stunting among children whose mothers were overweight and obese ${ }^{(6)}$. Although there has been a notable decline in childhood undernutrition levels in Kenya ${ }^{(9,10)}$, stunting in children remains higher than the WHO cut-off for public health significance of $<20 \%{ }^{(11)}$. On the other hand, the prevalence of obesity and overweight (BMI $>25 \mathrm{~kg} / \mathrm{m}^{2}$ ) in Kenyan women is steadily increasing from $23 \%$ in $2003^{(12)}$ to $25 \%$ in 2008-2009 $9^{(13)}$ and $38 \%$ in 2014 ${ }^{(10)}$. Fig. 1 demonstrates a conceptual framework for the double burden of over- and undernutrition adapted from the WHO Regional Office for South-East Asia ${ }^{(14)}$ based on the UNICEF 1997 causes of malnutrition $^{(15)}$. Our framework is also informed by other authors $^{(4,16-19)}$. This framework gives a background that underpins the causes of double burden of mother-child pairs of over- and undernutrition. The underlying determinants are of interest because a mother and her child's nutrition are closely related. Garret $\&$ Ruel $^{(8)}$ hypothesised that in countries experiencing the nutrition transition, families may be able to purchase food but not in the right nutrient balance ${ }^{(8)}$.
Affordable foods may be those that contain high energy with low concentration of other nutrients. On one hand, overweight and obesity among women results from a low-quality diet which is characterised by processed energy-dense foods with high sugar, salt and saturated fat and often low in fibre ${ }^{(20,21)}$. On the other hand, inadequate care, poor-quality diets and inadequate access to clean water and sanitation underpin the suboptimal nutrition outcomes in the children $^{(22)}$. Micronutrient deficiencies in adults have also been associated with overweight and obesity ${ }^{(23)}$. In addition, a monotonous diet low in animal-source foods leads to micronutrient deficiencies which are associated with child stunting.

Undernutrition or overnutrition has health and development long-term consequences at individual and wider-society levels. Impaired cognitive development and poor school performance resulting from childhood undernutrition affect economic productivity in adult life ${ }^{(16)}$. Overweight and obesity lead to impaired economic productivity and increase expenditure on health care ${ }^{(24)}$. Both outcomes derail the public health care system and strain individual and household resources. It is important to quantify the double burden of malnutrition in Kenya to inform appropriate intervention strategies. Using the 2014 Kenya Demographic and Health Survey (KDHS) nationally representative population data, we present evidence on the magnitude and determinants of the mother-child pairs of double burden of malnutrition. The DHS is a highquality standardised periodic survey providing data for a wide range of population, health and nutrition indicators. The DHS 
reports mainly present descriptive data and do not include detailed analysis associations or predictors of health and other developmental outcomes. In the present study, we conducted further analysis to present evidence on the magnitude and determinants of the mother-child pairs of double burden of malnutrition. A mother-child pair of double burden of malnutrition in this study was defined as an overweight or obese mother whose child is stunted, underweight or wasted.

\section{Methods}

\section{Dataset}

The KDHS 2014 dataset was used for this analysis. The KDHS 2014 utilised multistage stratified cluster sampling methodology ${ }^{(10)}$. Samples of households within clusters (enumeration areas) were selected based on a master sampling frame of the Fifth National Sample Survey and Evaluations Programme (NASSEP V). In the NASSEP V, each of the forty-seven administrative counties in Kenya was stratified into rural and urban strata except for Nairobi and Mombasa counties that have only urban areas. Based on this sampling frame, a total of ninety-two sampling strata were utilised with 1612 clusters (Fig. 2). In the first level of selection all the 1612 clusters were included with equal probability from the NASSEP V frame. In the second stage of selection, a households listing was used as the sampling frame in which twenty-five households were selected from each cluster ${ }^{(10)}$. Residents in the selected households participated in the survey. Urban areas were oversampled, and the present analysis was based on weighted data to cater for the different sample proportions. A total of 39679 households were selected for the 2014 survey and 36430 were interviewed, yielding a $99 \%$ response rate. A total of 14741 women were interviewed in the households selected for the full household questionnaires which included measurements of height and weight for children less than 5 years and women. For our analysis, a weighted subsample of 7830 mother-child pairs was included based on the DHS children's recode data file ${ }^{(25)}$. The analysis included children under 5 years of age whose weight and height/length measurements were taken and whose mothers were interviewed and had their weights and heights measured. Sample weights were calculated based on the DHS sampling weights methodology ${ }^{(26)}$.

\section{Anthropometric measurements and nutritional status}

In the KDHS, measurement of height and weight were taken for children born since January 2009 who were listed in the household questionnaire. Child weight was taken with electronic SECA digital scales to the nearest $100 \mathrm{~g}$ while child height was measured using Shorr height/length boards made by the Shorr productions ${ }^{(10)}$. The SECA digital weight scales used in the DHS survey were made with guidance from $\mathrm{UNICEF}^{(10)}$. Recumbent length was measured lying down for children younger than 24 months and for those with unknown age and less than $87 \mathrm{~cm}$. Child nutritional status was determined using the WHO 2006 reference standard to

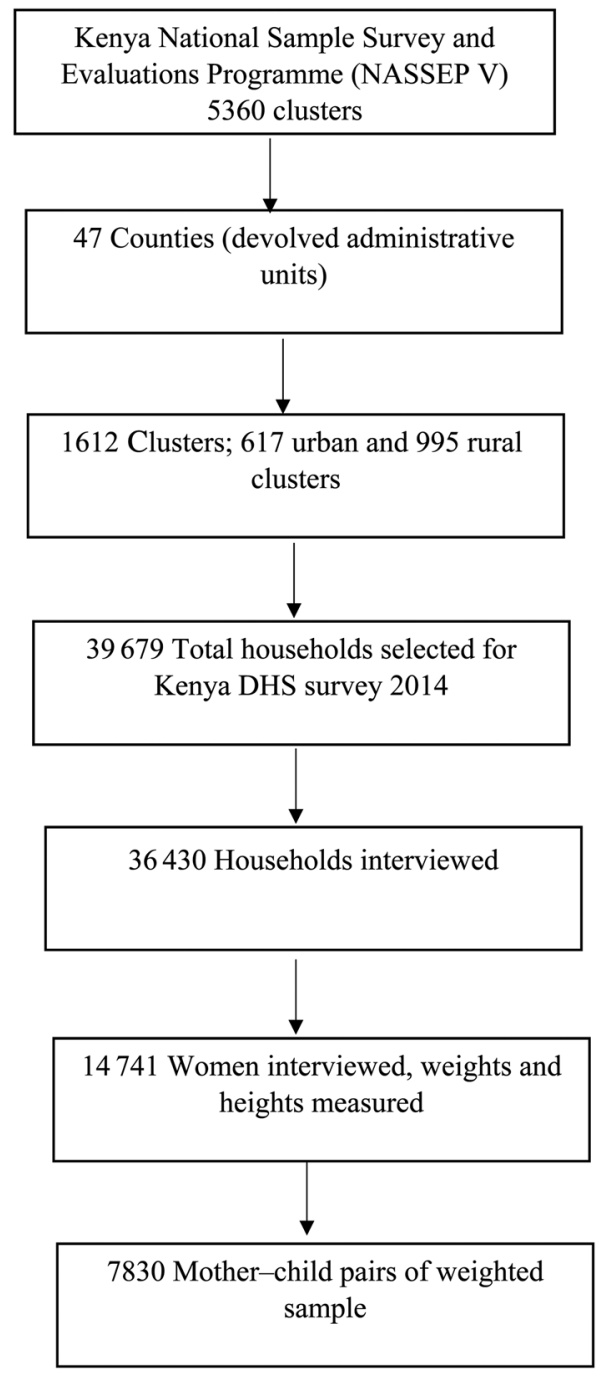

Fig. 2. Flowchart describing the sampling and sample selection procedure for the analysis of the double burden of malnutrition in Kenya. DHS, Demographic and Health Survey.

calculate standard deviation $z$-scores. Childhood undernutrition was defined as height-for-age, weight-for-age and weight-for-height $z$-scores below -2 standard deviations from the WHO reference standard, being stunting, underweight and wasting, respectively. Childhood overweight was defined as weight-for-height $>+2$ standard deviation $z$-score. Children were excluded if their anthropometric measurements were invalid with height-for-age or weight-for-age $z$-scores below -6 standard deviation or above +6 standard deviation or with weight-for-height below -5 or above +5 standard deviations from the WHO reference standard ${ }^{(27)}$. Other child characteristics included in the analysis were age, sex, birth order and recent illness such as fever, diarrhoea or cough. Maternal weight and height were measured with SECA electronic scales made by the Shorr productions and Shorr height boards. BMI was derived as weight in kilograms divided by the square of height in metres.

Mothers' nutritional status was defined as: BMI $<18.5 \mathrm{~kg} / \mathrm{m}^{2}$, underweight; BMI $18.5-24.9 \mathrm{~kg} / \mathrm{m}^{2}$, normal; BMI $\geq 25$ $29.9 \mathrm{~kg} / \mathrm{m}^{2}$, overweight; BMI $\geq 30 \mathrm{~kg} / \mathrm{m}^{2}$, obese. Pregnant 
women, women with a delivery within 2 months before the survey and those with invalid anthropometric measurements were excluded from this analysis. Mother-child pairs of double burden of over- and undernutrition was defined as an overweight or obese mother with a stunted, underweight or wasted child. Other maternal characteristics included in the analysis were age, maternal education, parity, marital status and work status.

\section{Household characteristics}

Household wealth index, residence (rural or urban), source of drinking water and toilet facilities were included as independent characteristics in this analysis. The wealth index in the DHS is a proxy for household long-term living standards. The list of household assets and services used to calculate the wealth index in the DHS includes the type of materials used in house construction, water supply and sanitation facilities, electricity, radio, television, refrigerator, watch, type of vehicle, furniture, ownership of agriculture land and farm animals, cooking fuel, bank account, appliance items such as blender, water heater, camera or electronic generators ${ }^{(28)}$. The index is generated through principal components analysis that assigns a factor score for each asset. The resulting asset scores are standardised in a normal distribution ${ }^{(29)}$. The wealth index is grouped into five categories as poorest, poorer, middle, richer and richest households. Improved water sources are those adequately protected from contamination ${ }^{(30)}$. Improved water sources in the DHS include water from a piped water system, protected wells or springs, bottled or harvested rain water. Non-improved water sources are unprotected wells, springs, rivers, ponds, lakes and dams. Improved sanitation facilities were considered as those that separated human faecal matter from human contact hygienically ${ }^{(30)}$. In this study, improved sanitation facilities were flush toilets and ventilated and improved pit (VIP) latrines. Traditional pit latrines were categorised as non-improved sanitation facilities.

\section{Statistical analysis}

Data were analysed using Stata software version 12.0 for Windows at the descriptive, bivariate and multivariate levels. Descriptive statistics reported frequencies and percentages for categorical data while means and standard errors were reported for continuous data. Sampling weights were applied to account for the survey sampling design used in the DHS. Bivariate logistic regression was used to check for relationships between the mother-child pairs and the household, maternal and child characteristics. Associations at bivariate and predictors at multivariate levels were reported in terms of OR and $95 \%$ CI. Statistical significance was considered at $P<0.05$.

\section{Results}

We analysed a weighted total of 7830 mother-child pairs. The mothers were aged between 15 and 49 years and the children were under the age of 5 years. The mothers' mean age was 29.0 (95\% CI $28 \cdot 7,29 \cdot 2)$ years. Slightly more than half of the mothers were aged between 20 and 30 years (53.3\%). A higher proportion resided in the rural areas $(65.1 \%)$ and had access to improved water sources $(64.8 \%)$ (Supplementary Table S1). The mothers' mean BMI was $23.4\left(95 \%\right.$ CI 23.2-23.6) $\mathrm{kg} / \mathrm{m}^{2}$. The prevalence of overweight (BMI 25 to $29.9 \mathrm{~kg} / \mathrm{m}^{2}$ ) among the mothers was $21.5 \%$ while $8.4 \%$ were obese (BMI $\geq 30 \mathrm{~kg} / \mathrm{m}^{2}$ ) (Supplementary Table S1). The combined prevalence of overweight and obesity amongst the women was $26 \%$. The mean height-for-age, weight-for-age and weight-for-height $z$-scores among the children were $-1 \cdot 14,-0.63$ and 0.02 , respectively. About one-quarter $(26.3 \%)$ of the children were stunted, $12.8 \%$ were underweight and $5.1 \%$ were wasted. Less than $5 \%(3.5 \%)$ of children were overweight. Stunting levels were higher in boys $(28.7 \%$ ) than girls $(21.6 \%)$. A quarter of the children had fever $(25.1 \%), 16.1 \%$ had diarrhoea and $38.2 \%$ had cough as a recent illness preceding the survey (Supplementary Table S1).

\section{Mother-child pairs of double burden of malnutrition}

A mother-child pair of double burden was defined as an overweight or obese mother (BMI $\geq 25 \mathrm{~kg} / \mathrm{m}^{2}$ ) with a stunted, underweight or wasted child. The weighted total number of mothers with overweight or obesity was 2034 (26.0\%). Out of these mothers, 571 had children who were either stunted, underweight or wasted distributed as follows: overweight/ obese mother-stunted child pairs, $20 \%$; overweight/obese mother-underweight child pairs, 5.4\%; overweight/obese mother-wasted child, 3.1\% (Table 1). Overweight/obese mother-stunted child pairs occurred more in the urban areas $(17.9 \%)$ and among the richer $(14.6 \%)$ and richest $(21.5 \%)$ wealth quintile households. Similarly, the proportion of overweight/obese mother-stunted child pairs was higher among the mothers with post-secondary (college) level of education $(15 \cdot 17 \%)$ (Table 1$)$.

\section{Mother-child pairs of double burden of malnutrition by household, maternal and child characteristics}

Overweight/obese mother-stunted child pairs. Overweight/ obese mother-stunted child pairs were less likely to occur in rural areas compared with the urban areas (adjusted OR $(\mathrm{aOR})=0.54 ; 95 \%$ CI $0.38,0.78 ; P=0.001)$. Overweight $/$ obese mother-stunted child pairs were less likely to occur in the poorest $(\mathrm{aOR}=0.42 ; 95 \%$ CI $0.22,0.82 ; P=0.01)$, poorer $(\mathrm{aOR}=0.38 ; 95 \% \mathrm{CI} 0.21,0.68 ; P<0.001)$ and middle $(\mathrm{aOR}=0.40 ; 95 \%$ CI $0.23,0.69 ; P<0.001)$ wealth quintile households compared with the richest wealth quintile households. Overweight/obese mother-stunted child pairs were more likely to occur if the child was male (aOR $=0.57 ; 95 \%$ CI $0.43,0.76 ; P<0.0001)$ compared with the mother-child pairs with female children. The risk of having an overweight/obese mother-stunted child pair was highest among the children aged 13-24 months (aOR = 6.81; $95 \%$ CI 2.63, 17.64; $P<0.0001)$ compared with children less than 6 months old (Table 2). 
https://doi.org/10.1017/jns.2019.39 Published online by Cambridge University Press

Table 1. Distribution of mother-child pairs of double burden of malnutrition by household, maternal and child characteristics, Kenya Demographic and Health Survey, 2014 (Numbers of pairs and percentages)

\begin{tabular}{|c|c|c|c|c|c|c|c|c|}
\hline \multirow[b]{2}{*}{ Background variable } & \multirow[b]{2}{*}{ Category } & \multicolumn{2}{|c|}{$\begin{array}{l}\text { Overweight/obese } \\
\text { mother-stunted child }\end{array}$} & \multicolumn{2}{|c|}{$\begin{array}{l}\text { Overweight/obese } \\
\text { mother-underweight child }\end{array}$} & \multicolumn{2}{|c|}{$\begin{array}{l}\text { Overweight/obese } \\
\text { mother-wasted child }\end{array}$} & \multirow{2}{*}{$\begin{array}{c}\text { Number of } \\
\text { overweight/obese } \\
\text { women } \\
\left(\mathrm{BMl} \geq 25 \mathrm{~kg} / \mathrm{m}^{2}\right)\end{array}$} \\
\hline & & $n$ & Weighted \% & $n$ & Weighted \% & $n$ & Weighted \% & \\
\hline \multicolumn{9}{|l|}{ Household characteristics } \\
\hline \multirow[t]{2}{*}{ Residence } & Urban & 177 & 17.93 & 52 & 5.4 & 30 & $2 \cdot 8$ & 1054 \\
\hline & Rural & 221 & 7.85 & 58 & 1.72 & 33 & 0.78 & 980 \\
\hline \multirow[t]{5}{*}{ Wealth quintile } & Poorest & 92 & 7.74 & 31 & 1.92 & 16 & 0.69 & 289 \\
\hline & Poorer & 71 & 7.5 & 15 & 1.32 & 4 & 0.32 & 326 \\
\hline & Middle & 68 & $7 \cdot 8$ & 22 & $2 \cdot 82$ & 16 & 1.92 & 362 \\
\hline & Richer & 82 & 14.64 & 15 & $2 \cdot 61$ & 9 & 1.34 & 463 \\
\hline & Richest & 85 & 21.52 & 27 & $7 \cdot 26$ & 18 & 3.81 & 594 \\
\hline \multirow{2}{*}{ Water source } & Improved water source & 243 & $12 \cdot 4$ & 75 & 3.51 & 52 & 1.97 & 1407 \\
\hline & Non-improved water source & 145 & 9.23 & 33 & 1.96 & 10 & 0.51 & 568 \\
\hline \multirow{2}{*}{ Toilet facilities ( $n$ 392) } & Improved toilet facilities & 191 & 14.28 & 53 & 4.26 & 33 & 2.47 & 1140 \\
\hline & Non-improved toilet facilities & 201 & 8.93 & 56 & 1.88 & 29 & 0.62 & 859 \\
\hline \multicolumn{9}{|l|}{ Maternal characteristics } \\
\hline \multirow{4}{*}{ Age categories } & $<20$ years & 9 & 4.65 & 2 & 0.93 & 2 & 0.9 & 33 \\
\hline & $20-30$ years & 177 & 9.87 & 44 & $2 \cdot 15$ & 29 & $1 \cdot 16$ & 918 \\
\hline & $30-40$ years & 163 & 13.39 & 52 & 4.33 & 27 & 1.82 & 874 \\
\hline & $40+$ years & 49 & $15 \cdot 32$ & 12 & $2 \cdot 83$ & 5 & 1.23 & 209 \\
\hline \multirow[t]{4}{*}{ Education } & None & 55 & 7.75 & 26 & 3.06 & 19 & $1 \cdot 31$ & 208 \\
\hline & Completed primary & 226 & 10.92 & 61 & 2.94 & 25 & 1.07 & 1031 \\
\hline & Completed secondary & 86 & 11.92 & 16 & 1.98 & 16 & 1.91 & 542 \\
\hline & Post-secondary education & 31 & $15 \cdot 07$ & 7 & 4.55 & 3 & $2 \cdot 36$ & 253 \\
\hline \multirow{2}{*}{ Marital status } & Married & 356 & 11.82 & 101 & 3.12 & 55 & 1.36 & 1798 \\
\hline & Single & 42 & 7.68 & 9 & 1.37 & 8 & 1.34 & 236 \\
\hline \multirow[t]{2}{*}{ Work status ( $n$ 395) } & Working & 268 & $11 \cdot 24$ & 43 & $2 \cdot 9$ & 31 & $1 \cdot 32$ & 590 \\
\hline & Not working & 127 & 10.91 & 64 & 2.45 & 31 & 1.34 & 1432 \\
\hline \multirow[t]{3}{*}{ Number of children ever born } & 1 & 47 & 7.53 & 12 & 2.33 & 12 & 1.81 & 293 \\
\hline & $2-3$ & 154 & 12.65 & 36 & $2 \cdot 61$ & 16 & 1.09 & 885 \\
\hline & $4+$ & 197 & $11 \cdot 27$ & 62 & 3.23 & 35 & 1.41 & 856 \\
\hline \multicolumn{9}{|l|}{ Child characteristics } \\
\hline \multirow{5}{*}{ Age categories } & $<6$ months & 8 & 2.02 & 3 & 0.21 & 8 & 0.92 & 131 \\
\hline & 6-12 months & 26 & $5 \cdot 81$ & 11 & 2.53 & 6 & 0.79 & 268 \\
\hline & 13-24 months & 94 & 14.51 & 22 & 3.04 & 19 & 2.3 & 366 \\
\hline & $25-36$ months & 101 & 13.58 & 21 & $2 \cdot 41$ & 9 & $1 \cdot 18$ & 443 \\
\hline & $37-59$ months & 169 & $12 \cdot 81$ & 53 & 3.69 & 21 & 1.25 & 826 \\
\hline \multirow[t]{2}{*}{ Sex } & Male & 235 & 13.63 & 65 & 3.39 & 35 & 1.57 & 1029 \\
\hline & Female & 163 & 8.65 & 45 & $2 \cdot 24$ & 28 & $1 \cdot 14$ & 1005 \\
\hline Birth order & 1 & 66 & 7.62 & 18 & 2.06 & 13 & 1.34 & 391 \\
\hline & $2-3$ & 155 & 12.94 & 38 & 2.99 & 16 & $1 \cdot 14$ & 859 \\
\hline & $4+$ & 177 & 11.62 & 54 & 3.15 & 34 & 1.58 & 784 \\
\hline Recent illness & & & & & & & & \\
\hline Fever & No & 303 & 11.68 & 83 & 3.02 & 43 & 1.44 & 1558 \\
\hline & Yes & 95 & 9.66 & 27 & 2.29 & 20 & 1.15 & 474 \\
\hline & No & 335 & $10 \cdot 71$ & 93 & $2 \cdot 77$ & 51 & 1.33 & 1749 \\
\hline Diarrhoea & Yes & 63 & $13 \cdot 28$ & 17 & 3.1 & 12 & 1.53 & 284 \\
\hline & No & 248 & 11.29 & 82 & 3.79 & 46 & 1.77 & 1260 \\
\hline Cough & Yes & 150 & 10.89 & 28 & 1.32 & 17 & 0.73 & 772 \\
\hline Total & & 398 & 19.57 & 110 & 5.41 & 63 & $3 \cdot 10$ & 2034 \\
\hline
\end{tabular}


Table 2. Predictors of overweight/obese mother-stunted child pairs of double burden of malnutrition by household, maternal and child characteristics, Kenya Demographic and Health Survey, 2014*

(Unadjusted odds ratios (uOR), adjusted odds ratios (aOR) and $95 \%$ confidence intervals)

\begin{tabular}{|c|c|c|c|c|c|c|c|}
\hline \multirow[b]{2}{*}{ Background variable } & \multirow[b]{2}{*}{ Category } & \multicolumn{6}{|c|}{ Overweight/obese mother-stunted child } \\
\hline & & uOR & $95 \% \mathrm{Cl}$ & $P$ & $\mathrm{aOR}$ & $95 \% \mathrm{Cl}$ & $P$ \\
\hline \multicolumn{8}{|c|}{ Household characteristics } \\
\hline \multirow[t]{2}{*}{ Residence } & Urban & \multicolumn{2}{|c|}{ Reference } & \multicolumn{4}{|c|}{ Reference } \\
\hline & Rural & 0.39 & $0.28,0.54$ & $<0.0001$ & 0.54 & $0.38,0.78$ & 0.001 \\
\hline \multirow[t]{5}{*}{ Wealth quintile } & Poorest & 0.31 & $0.18,0.51$ & $<0.0001$ & 0.42 & $0.22,0.82$ & 0.01 \\
\hline & Poorer & 0.30 & $0.18,0.49$ & $<0.0001$ & 0.38 & $0.21,0.68$ & 0.001 \\
\hline & Middle & 0.31 & $0.19,0.50$ & $<0.0001$ & 0.40 & $0.23,0.69$ & 0.001 \\
\hline & Richer & 0.63 & $0.37,1.05$ & 0.074 & 0.76 & $0.43,1.32$ & 0.328 \\
\hline & Richest & \multicolumn{2}{|c|}{ Reference } & \multicolumn{4}{|c|}{ Reference } \\
\hline \multirow[t]{2}{*}{ Water source } & Improved water source & \multicolumn{2}{|c|}{ Reference } & \multicolumn{4}{|c|}{ Reference } \\
\hline & Non-improved water source & 0.72 & $0.52,0.99$ & 0.044 & $1 \cdot 10$ & $0.78,1.56$ & 0.583 \\
\hline \multicolumn{8}{|l|}{ Toilet facilities } \\
\hline & Improved toilet facilities & \multicolumn{2}{|c|}{ Reference } & \multicolumn{4}{|c|}{ Reference } \\
\hline & Non-improved toilet facilities & 0.59 & $0.43,0.80$ & 0.001 & 1.04 & $0.73,1.48$ & 0.837 \\
\hline \multicolumn{8}{|c|}{ Maternal characteristics } \\
\hline & $<20$ years & 0.27 & $0.11,0.64$ & 0.003 & 0.73 & $0.24,2 \cdot 20$ & 0.577 \\
\hline & $20-30$ & 0.61 & $0.37,0.98$ & 0.04 & 0.66 & $0.36,1.19$ & 0.164 \\
\hline & $30-40$ & 0.85 & $0.53,1.37$ & 0.511 & 0.77 & $0.46,1.27$ & 0.305 \\
\hline & $40+$ & & ference & & & erence & \\
\hline Education & & & & & & & \\
\hline & None & 0.47 & $0.25,0.90$ & 0.022 & 0.82 & $0.38,1.78$ & 0.632 \\
\hline & Completed primary & 0.69 & $0.41,1.16$ & 0.162 & 1.15 & $0.61,2.16$ & 0.664 \\
\hline & Completed secondary & 0.76 & $0.42,1.40$ & 0.381 & 0.96 & $0.49,1.90$ & 0.912 \\
\hline & Post-secondary education & & ference & & & erence & \\
\hline Marital status & & & & & & & \\
\hline & Married & & erence & & & erence & \\
\hline & Single & 0.62 & $0.40,0.96$ & 0.032 & 0.79 & $0.49,1.28$ & 0.344 \\
\hline Work status & & & & & & & \\
\hline & Working & 1.03 & $0.72,1.48$ & 0.855 & & & \\
\hline & Not working & & ference & & & & \\
\hline Number of children & & & & & & & \\
\hline & 1 & & erence & & & erence & \\
\hline & $2-3$ & 1.78 & $1.11,2.85$ & 0.016 & 1.01 & $0.40,2.58$ & 0.982 \\
\hline & $4+$ & 1.56 & $0.99,2.45$ & 0.053 & 0.81 & $0.24,2.73$ & 0.736 \\
\hline Child characteristics & & & & & & & \\
\hline Age categories & $<6$ months & & ference & & & erence & \\
\hline & 6-12 months & 2.99 & $1.15,7.79$ & 0.025 & 2.66 & $0.99,7.14$ & 0.052 \\
\hline & 13-24 months & 8.23 & $3.23,20.95$ & $<0.0001$ & $6 \cdot 81$ & $2.63,17.64$ & $<0.0001$ \\
\hline & 25-36 months & 7.62 & $3 \cdot 19,18 \cdot 21$ & $<0.0001$ & 5.84 & $2.36,14.43$ & $<0.0001$ \\
\hline & 37-59 months & $7 \cdot 12$ & $2.98,17.03$ & $<0.0001$ & 6.06 & $2 \cdot 42,15 \cdot 16$ & $<0.0001$ \\
\hline Sex & & & & & & & \\
\hline & Male & & ference & & & erence & \\
\hline & Female & 0.60 & $0.46,0.79$ & $<0.0001$ & 0.57 & $0.43,0.76$ & $<0.0001$ \\
\hline Birth order & & & & & & & \\
\hline & 1 & & ference & & & erence & \\
\hline & $2-3$ & 1.80 & $1 \cdot 24,2 \cdot 62$ & 0.002 & 1.82 & $0.87,3.82$ & 0.113 \\
\hline & $4+$ & 1.59 & $1.06,2.40$ & 0.025 & 2.50 & $0.87,7.19$ & 0.088 \\
\hline Recent illness & & & & & & & \\
\hline Fever & No & & ference & & & erence & \\
\hline & Yes & 0.81 & $0.59,1.11$ & 0.189 & 0.81 & $0.60,1.11$ & 0.188 \\
\hline Diarrhoea & No & & ference & & & & \\
\hline & Yes & 1.27 & $0.81,2.01$ & 0.292 & & & \\
\hline Cough & No & & ference & & & & \\
\hline & Yes & 0.96 & $0.70,1.32$ & 0.806 & & & \\
\hline
\end{tabular}

* Bivariate logistic regression was used to report associations by use of uOR. Variables that had a $P<0.2$ in the bivariate model were controlled for in the multivariate model (residence, wealth quintile, water source, toilet facilities, maternal age, maternal education, marital status, number of children, child age, child sex, child birth order, fever). Multivariate logistic regression was used to obtain the independent determinants and reported by use of aOR.

Overweight/obese mother-underweight child pairs. The odds of overweight/obese mother-underweight child pairs were lower among mother-child pairs living in rural areas than those in the urban areas $(\mathrm{aOR}=0.43 ; 95 \% \mathrm{CI} 0.23$,
$0.79 ; P=0.007)$. At the bivariate level of analysis, household wealth was significantly associated with overweight/obese mother-underweight child pairs. The poorest, poorer, middle and richer wealth quintiles had lower odds of 
Table 3. Predictors of overweight/obese mother-underweight child pairs of double burden of malnutrition by household, maternal and child characteristics, Kenya Demographic and Health Survey, 2014*

(Unadjusted odds ratios (UOR), adjusted odds ratios (aOR) and $95 \%$ confidence intervals)

\begin{tabular}{|c|c|c|c|c|c|c|c|}
\hline \multirow[b]{2}{*}{ Background variable } & \multirow[b]{2}{*}{ Category } & \multicolumn{6}{|c|}{ Overweight/obese mother-underweight child } \\
\hline & & uOR & $95 \% \mathrm{Cl}$ & $P$ & $\mathrm{aOR}$ & $95 \% \mathrm{Cl}$ & $P$ \\
\hline \multicolumn{8}{|c|}{ Household characteristics } \\
\hline \multirow[t]{2}{*}{ Residence } & Urban & \multicolumn{2}{|c|}{ Reference } & \multicolumn{4}{|c|}{ Reference } \\
\hline & Rural & 0.31 & $0.18,0.52$ & $<0.0001$ & 0.43 & $0.23,0.79$ & 0.007 \\
\hline \multirow[t]{5}{*}{ Wealth quintile } & Poorest & 0.25 & $0.12,0.52$ & $<0.0001$ & 0.68 & $0.25,1.87$ & 0.46 \\
\hline & Poorer & 0.17 & $0.07,0.39$ & $<0.0001$ & 0.44 & $0.17,1.14$ & 0.09 \\
\hline & Middle & 0.37 & $0.16,0.84$ & 0.017 & 0.84 & $0.33,2.18$ & 0.724 \\
\hline & Richer & 0.34 & $0.14,0.84$ & 0.019 & 0.56 & $0.23,1.40$ & 0.213 \\
\hline & Richest & \multicolumn{2}{|c|}{ Reference } & \multicolumn{4}{|c|}{ Reference } \\
\hline \multirow[t]{2}{*}{ Water source } & Improved water source & \multicolumn{2}{|c|}{ Reference } & \multicolumn{4}{|c|}{ Reference } \\
\hline & Non-improved water source & 0.55 & $0.32,0.95$ & 0.032 & 0.89 & $0.49,1.61$ & 0.7 \\
\hline \multicolumn{8}{|l|}{ Toilet facilities } \\
\hline & Improved toilet facilities & \multicolumn{2}{|c|}{ Reference } & \multicolumn{4}{|c|}{ Reference } \\
\hline & Non-improved toilet facilities & 0.43 & $0.26,0.72$ & 0.001 & 0.71 & $0.43,1.17$ & 0.182 \\
\hline \multicolumn{8}{|c|}{ Maternal characteristics } \\
\hline & $<20$ years & 0.32 & $0.06,1.83$ & 0.202 & 0.43 & $0.07,2.65$ & 0.36 \\
\hline & 20-30 years & 0.75 & $0.33,1.71$ & 0.497 & 0.55 & $0.24,1.26$ & 0.156 \\
\hline & $30-40$ years & 1.55 & $0.69,3.51$ & 0.29 & 1.08 & $0.48,2.40$ & 0.857 \\
\hline & $40+$ years & & erence & & & erence & \\
\hline Education & & & & & & & \\
\hline & None & 0.66 & $0 \cdot 20,2 \cdot 13$ & 0.489 & & & \\
\hline & Completed primary & 0.64 & $0.21,1.91$ & 0.419 & & & \\
\hline & Completed secondary & 0.42 & $0.13,1.41$ & 0.162 & & & \\
\hline & Post-secondary education & & erence & & & & \\
\hline Marital status & & & & & & & \\
\hline & Married & & erence & & & & \\
\hline & Single & 0.43 & $0.18,1.02$ & 0.055 & & & \\
\hline Work status & & & & & & & \\
\hline & Working & $1 \cdot 19$ & $0.65,2.177$ & 0.578 & & & \\
\hline & Not working & & erence & & & & \\
\hline Number of children & & & & & & & \\
\hline & 1 & & erence & & & & \\
\hline & $2-3$ & 1.12 & $0.50,2.52$ & 0.779 & & & \\
\hline & $4+$ & 1.40 & $0.64,3.08$ & 0.403 & & & \\
\hline Child characteristics & & & & & & & \\
\hline Age categories & & & & & & & \\
\hline & $<6$ months & & erence & & & erence & \\
\hline & 6-12 months & $12 \cdot 07$ & $2 \cdot 15,67 \cdot 62$ & 0.005 & 12.04 & $2 \cdot 15,67 \cdot 30$ & 0.005 \\
\hline & 13-24 months & 14.57 & $2.88,73.83$ & 0.001 & 13.99 & $2.69,72.69$ & 0.002 \\
\hline & 25-36 months & 11.48 & $2 \cdot 27,58 \cdot 12$ & 0.003 & $10 \cdot 40$ & $2 \cdot 03,53 \cdot 19$ & 0.005 \\
\hline & $37-59$ months & $17 \cdot 80$ & $3.66,86.53$ & $<0.0001$ & 15.04 & $3.06,73.84$ & 0.001 \\
\hline Sex & & & & & & & \\
\hline & Male & & erence & & & erence & \\
\hline & Female & 0.65 & $0.40,1.07$ & 0.091 & 0.68 & $0.42,1 \cdot 11$ & 0.124 \\
\hline Birth order & & & & & & & \\
\hline & 1 & & erence & & & & \\
\hline & $2-3$ & 1.47 & $0.70,3.06$ & 0.307 & & & \\
\hline & $4+$ & 1.55 & $0.74,3.23$ & 0.244 & & & \\
\hline Recent illness & & & & & & & \\
\hline Fever & No & & erence & & & & \\
\hline & Yes & 0.76 & $0.41,1.39$ & 0.367 & & & \\
\hline Diarrhoea & No & & erence & & & & \\
\hline & Yes & 1.12 & $0.55,2.30$ & 0.753 & & & \\
\hline Cough & No & & erence & & & erence & \\
\hline & Yes & 0.34 & $0.19,0.60$ & $<0.0001$ & 0.36 & $0.20,0.64$ & $<0.0001$ \\
\hline
\end{tabular}

* Bivariate logistic regression was used to report associations by use of uOR. Variables that had a $P<0.2$ in the bivariate model were controlled for in the multivariate model (residence, wealth quintile, water source, toilet facilities, cough, maternal age, child age and child sex). Multivariate logistic regression was used to obtain the independent determinants and reported by use of aOR.

observing overweight/obese mother-underweight child pairs (Table 3). However, the results were not statistically significant in the multivariate adjusted model. The risk of having an overweight/obese mother-underweight child pair increased with child's age with the highest odds observed in the 37- to 59-month-old compared with less than 6-month-old children $(\mathrm{aOR}=15 \cdot 04 ; 95 \%$ CI 3.06, 73.84; $P=0.001)$. Reported childhood diarrhoea in the recent past was not significantly 
Table 4. Predictors of overweight/obese-wasted child pairs of double burden of malnutrition by household, maternal and child characteristics, Kenya Demographic and Health Survey, 2014*

(Unadjusted odds ratios (uOR), adjusted odds ratios (aOR) and $95 \%$ confidence intervals)

\begin{tabular}{|c|c|c|c|c|c|c|c|}
\hline \multirow[b]{2}{*}{ Background variable } & \multirow[b]{2}{*}{ Category } & \multicolumn{6}{|c|}{ Overweight/obese mother-wasted child } \\
\hline & & uOR & $95 \% \mathrm{Cl}$ & $P$ & aOR & $95 \% \mathrm{Cl}$ & $P$ \\
\hline \multicolumn{8}{|c|}{ Household characteristics } \\
\hline \multirow[t]{2}{*}{ Residence } & Urban & \multicolumn{2}{|c|}{ Reference } & \multicolumn{4}{|c|}{ Reference } \\
\hline & Rural & 0.27 & $0.14,0.522$ & $<0.0001$ & 0.53 & $0.23,1.26$ & 0.152 \\
\hline \multirow[t]{5}{*}{ Wealth quintile } & Poorest & 0.17 & $0.07,0.422$ & $<0.0001$ & 0.83 & $0.22,3.13$ & 0.778 \\
\hline & Poorer & 0.08 & $0.02,0.26$ & $<0.0001$ & 0.27 & $0.07,1.06$ & 0.06 \\
\hline & Middle & 0.49 & $0.19,1.29$ & 0.151 & 1.33 & $0.35,5.01$ & 0.677 \\
\hline & Richer & 0.34 & $0.13,0.93$ & 0.036 & 0.49 & $0.17,1.38$ & 0.179 \\
\hline & Richest & \multicolumn{2}{|c|}{ Reference } & \multicolumn{4}{|c|}{ Reference } \\
\hline \multirow[t]{2}{*}{ Water source } & Improved water source & \multicolumn{2}{|c|}{ Reference } & \multicolumn{4}{|c|}{ Reference } \\
\hline & Non-improved water source & 0.25 & $0.11,0.58$ & 0.001 & 0.38 & $0.16,0.90$ & 0.027 \\
\hline \multicolumn{8}{|l|}{ Toilet facilities } \\
\hline & Improved toilet facilities & \multicolumn{2}{|c|}{ Reference } & \multicolumn{4}{|c|}{ Reference } \\
\hline & Non-improved toilet facilities & 0.25 & $0.13,0.48$ & $<0.0001$ & 0.37 & $0.17,0.79$ & 0.011 \\
\hline \multicolumn{8}{|c|}{ Maternal characteristics } \\
\hline & & & & & & & \\
\hline & $<20$ years & 0.73 & $0.11,4.87$ & 0.749 & & & \\
\hline & $20-30$ years & 0.94 & $0.29,3.03$ & 0.918 & & & \\
\hline & $30-40$ years & 1.49 & $0.45,4.94$ & 0.509 & & & \\
\hline & $40+$ years & & erence & & & & \\
\hline Education & & & & & & & \\
\hline & None & 0.55 & $0.13,2.38$ & 0.423 & & & \\
\hline & Completed primary & 0.45 & $0.11,1.91$ & 0.276 & & & \\
\hline & Completed secondary & 0.81 & $0.19,3.42$ & 0.773 & & & \\
\hline & Post-secondary education & & erence & & & & \\
\hline Marital status & & & & & & & \\
\hline & Married & & erence & & & & \\
\hline & Single & 0.98 & $0.38,2.50$ & 0.965 & & & \\
\hline Work status & & & & & & & \\
\hline & Working & 0.99 & $0.49,2.00$ & 0.971 & & & \\
\hline & Not working & & erence & & & & \\
\hline Number of children & & & & & & & \\
\hline & 1 & & erence & & & & \\
\hline & $2-3$ & 0.60 & $0.23,1.56$ & 0.297 & & & \\
\hline & $4+$ & 0.78 & $0.33,1.86$ & 0.571 & & & \\
\hline Child characteristics & & & & & & & \\
\hline Age categories & & & & & & & \\
\hline & $\begin{array}{l}<6 \text { months } \\
6-12 \text { months }\end{array}$ & 0.86 & $\begin{array}{l}\text { erence } \\
0.18,4.07\end{array}$ & 0.85 & & & \\
\hline & $13-24$ months & 2.54 & $0.88,7.35$ & 0.086 & & & \\
\hline & $25-36$ months & 1.28 & $0.39,4.21$ & 0.679 & & & \\
\hline & $37-59$ months & 1.37 & $0.45,4.18$ & 0.583 & & & \\
\hline Sex & & & & & & & \\
\hline & Male & & erence & & & & \\
\hline & Female & 0.72 & $0.36,1.43$ & 0.346 & & & \\
\hline Birth order & & & & & & & \\
\hline & 1 & & erence & & & & \\
\hline & $2-3$ & 0.85 & $0.33,2.19$ & 0.739 & & & \\
\hline & $4+$ & 1.18 & $0.50,2.82$ & 0.703 & & & \\
\hline Recent illness & & & & & & & \\
\hline Fever & No & & erence & & & & \\
\hline & Yes & 0.80 & $0.39,1.62$ & 0.53 & & & \\
\hline Diarrhoea & No & & erence & & & & \\
\hline & Yes & $1 \cdot 16$ & $0.48,2.77$ & 0.746 & & & \\
\hline Cough & No & & erence & & & rence & \\
\hline & Yes & 0.41 & $0.19,0.88$ & 0.022 & 0.41 & $0.19,0.90$ & 0.025 \\
\hline
\end{tabular}

* Bivariate logistic regression was used to report associations by use of uOR. Variables that had a $P<0.2$ in the bivariate model were controlled for in the multivariate model (residence, wealth quintile, water source, toilet facilities, cough). Multivariate logistic regression was used to obtain the independent determinants and reported by use of aOR.

associated with overweight/obese mother-underweight child pairs. Overweight/obese mother-underweight child pairs were less likely if the child had reported cough in the recent past compared with the children without a cough $(\mathrm{aOR}=$ 0.36; $95 \%$ CI 0.20, 0.64; $P<0 \cdot 001$ ) (Table 3).
Overweight/obese mother-wasted child pairs. Mother-child pairs with no access to an improved water source and improved toilet facilities were less likely to be an overweight/obese mother-wasted child pair compared with those having improved water sources and improved toilet 
facilities $(\mathrm{aOR}=0.38 ; 95 \%$ CI $0.16,0.19 ; P=0.027$; and aOR $=0.37 ; 95 \%$ CI $0.17,0.79 ; P=0.011$, respectively; Table 4). At the bivariate level of analysis, rural residents were less likely to have overweight/obese mother-wasted child pairs compared with urban residents $(\mathrm{uOR}=0 \cdot 27$; $95 \%$ CI $0.14,0.522 ; P<0.0001)$. Living in the poorest (uOR $=0.17 ; 95 \% \mathrm{CI} 0 \cdot 07,0.422 ; P<0.0001)$ and poorer (uOR $=0.08 ; \quad 95 \%$ CI $0.02, \quad 0.26 ; \quad P<0.0001$ ) wealth quintile households was less likely to be associated with overweight/obese mother-wasted child pairs compared with those living in the richest wealth quintile households. Overweight/obese mother-underweight child pairs were less likely if the child had reported a cough in the recent past compared with the children without a cough $(\mathrm{aOR}=$ $0 \cdot 41 ; 95 \%$ CI $0 \cdot 19,0 \cdot 90 ; P=0 \cdot 025$ ) (Table 4).

\section{Discussion}

We found that one-quarter of Kenyan children less than 5 years old were stunted, one in every ten children was underweight and less than $5 \%$ of children were wasted. We found a lower prevalence of childhood undernutrition from what has been previously reported in Kenya ${ }^{(10,12,13,31-33)}$. It is important to note the downward trend of childhood stunting in Kenya from $40 \%$ in 1993 to $35 \%$ in $2008-20009^{(10)}$, and a further decline to $26 \%$ in $2014^{(10)}$. Though the prevalence of childhood undernutrition is reducing in the country, the prevalence of adult overweight and obesity is steadily rising. Overweight and obesity among women of reproductive age increased from $23 \%$ in $2003^{(12)}$ to $25 \%$ in $2008-2009^{(13)}$, and $33 \%$ in $2014^{(10)}$. In this study, we found that one-quarter of women in Kenya are overweight or obese. The occurrence of overweight/obese mother-child undernutrition pairs is not an independent nutrition problem but rather a result of increasing prevalence of maternal overweight status in the population $^{(34)}$. This rapid rise in overweight/obesity in the adult population is faster than the decline in childhood undernutrition in Kenya.

We found notable levels of mother-child pairs of double burden of malnutrition in Kenya. The form of double burden prominent in the country was the overweight/obese mother-stunted child pair while the overweight/obese mother-underweight or wasted child occurred to a lesser extent. The overweight/obese mother-stunted phenomenon has been reported in a poor urban slum in Kenya ${ }^{(6)}$ and other parts of sub-Saharan Africa ${ }^{(35)}$. We found that living in urban areas, higher household wealth, and having a male child in the mother-child pair were significant predictors of the overweight/obese mother-stunted child pairs. At the bivariate level of analysis, access to improved water and sanitation facilities, lack of maternal education, younger maternal age, higher maternal parity and older child age, were associated with the overweight/obese mother-stunted child pairs. However, these associations were not statistically significant in the adjusted logistic regression model. Although maternal education is a known determinant of child nutrition outcomes $^{(9,32,36,37)}$, maternal education is positively correlated with household wealth ${ }^{(38)}$ and this correlation may explain the lack of statistical significance of maternal education for the double burden in the adjusted multivariate logistics model. Access to water and sanitation facilities are included in the DHS household wealth quintile variable. When included in the adjusted logistic model as independent variables, the water and sanitation facilities were not statistically significant determinants of the double burden.

In our present analysis, we found the proportion of overweight/obese mothers was equal for both the mothers of boys and the mothers of girls. However, the proportion of stunted boys was higher than that of girls. When the mother-child pair was considered, the overweight/obese-stunted child pairs more likely occurred among the mother-boy pairs than the mother-girl pairs. Our findings that the overweight/obese mother-stunted child pairs were more likely to occur if the child was male is not surprising noting that the sex differential in stunting rates has been consistently higher in boys than girls in Kenya ${ }^{(9)}$ and in similar settings ${ }^{(39-41)}$. The sex differential in stunting draws various biological and behavioural explanations. Biological explanations include the chromosomal differences between males and females that depict morbidity and mortality to be higher in male children than females ${ }^{(41,42)}$. Behavioural speculations about the sex differentials in stunting include aspects of selection bias, parental preferences of one sex over another, and cultural perceptions ${ }^{(41)}$. For instance, in Guatemala male infants have been reported as receiving complementary feeding younger than 6 months compared with girls because of cultural perceptions that infant boys were hungrier than girls and breastfeeding alone was not adequate for the boys $^{(43)}$. Early introduction to complementary feeding compromises the infant's immunity due to potential exposure to pathogens and increases the risk of infections ${ }^{(42)}$. Early complementary feeding reduces production of breast milk in the mother ${ }^{(42)}$ and may consequently results in more hungry children, increasing the need for more complementary foods. Breastfeeding is a vital component of the infant and young child feeding practices that promote healthy growth and development of children and reduces the incidence of undernutrition ${ }^{(44-46)}$. Poor breastfeeding patterns and suboptimal complementary feeding practices are widespread in low- and middle-income countries ${ }^{(47)}$. Suboptimal child feeding practices are associated with growth faltering and poor nutrition outcomes of young children ${ }^{(48-50)}$

In this study, the levels of child stunting and underweight were higher in rural areas while the prevalence of maternal overweight and obesity was higher in the urban areas (Supplementary Table S1). However, overweight/obese mother-stunted child pairs were more likely to occur in urban residence and higher wealth quintile households. Similar to our findings, Jehn \& Brewis reported that living in urban households increased the likelihood of mother overnutrition-child undernutrition pairs ${ }^{(51)}$. Our findings are an indication that maternal overweight in urban areas in Kenya is an important contributor to the urbanrural differentials of the double burden. Although childhood undernutrition is reducing in the country, maternal overnutrition and the double burden undermines the gains the country has made in the fight against undernutrition. In affluent urban areas, higher incomes encourage adoption of a more sedentary 
lifestyle which reduces physical activity, consequently lowering energy expenditure and resulting in overweight and obesity in adults $^{(52)}$. Household wealth in the DHS is measured using a composite variable based on a list of household possessions such as type of building materials for the house, access to clean drinking water and sanitation facilities, ownership of a car, electronics such as television, radio, refrigerator and telephones. Car ownership provides a more sedentary commute to work rather than on public transport that may encourage some walking to access a bus or train ride. Watching television is described as a proxy for seating time and was associated with overweight and obesity in Bangladesh ${ }^{(42)}$. A shift from manual labour to mechanised operations, from walking to motorised transportation, from physically labour-intensive economic activities to desk-bound work reduces energy expenditure. Sedentary lifestyle such as routinely sitting in a computer-based typical office chair is positively correlated with body weight ${ }^{(53)}$. Our study did not show any differences in the double burden of malnutrition between working and not working mothers. However, the work status as measured in this study included all forms of work and did not separate formal and informal types of work. In urban Kenyan cities such as Nairobi, it is common practice for working mothers in the formal sector to employ full-time domestic house helps who assist in household chores including child care, cleaning and meal preparation $^{(54)}$. Employing full-time domestic workers potentially compromises the quality of care that the child receives while the mother is out of home and away at work ${ }^{(55)}$. In addition, several studies have reported maternal employment as a persistent barrier to breastfeeding ${ }^{(56-60)}$.

The nutrition transition is associated with the emergence of a malnutrition double burden ${ }^{(2,51)}$. Although our analysis did not look at dietary intake variables, there is evidence of a nutrition transition in Kenya and Tanzania ${ }^{(61)}$, which is characterised by a shift in dietary patterns from traditional foods to the consumption of energy-dense and nutrient-poor foods. The nutrition transition is propagated by a dietary shift from plant-based highfibre diets to processed high-energy-dense foods ${ }^{(62)}$. The energydense foods lacking in other essential nutrients offer suboptimal nutrition for the children while contributing to weight gain in the mothers, thus the resultant double burden of malnutrition.

Our study had some strengths and limitations. Strengths include the use of nationally representative data to examine the double burden of maternal and child pairs that has not been reported previously at national scale. Although the analysis included a large sample of children and mothers, the subanalysis of the double burden for child underweight and wasting indicators was limited due to reduced underweight and wasting levels in the country. This analysis does not include dietary intake and physical activities which are important determinants of the double burden because such data are not included in the DHS. The cross-sectional nature of the data did not allow for an analysis of causal relationships for the double burden.

\section{Conclusion}

The double burden of overweight/obese mother-stunted child pairs is present in Kenya. The double burden dyads are more likely to occur in urban areas and in richer wealth quintile households. It is noteworthy that the boys have higher levels of stunting than girls and the overweight/obese mother-stunted child pairs are more likely to occur in the pairs with boys than those with girls. This phenomenon calls for further research to explore if there exist specific determinants of the mother-boy child dyad double burden. The double burden of malnutrition in the country presents a novel public health problem that complicates nutrition policy planning and interventions. This evidence is important to inform a policy and programming shift to address the mother-child dyads rather than attending to child undernutrition separate form maternal overnutrition.

\section{Ethics approval and consent to participate}

The ICF Institutional Review Board reviewed and approved the Kenya Demographic Health Survey and protocol. The ICF ensured that the study adhered to the US Department of Health and Human Services Regulations for the protection of human subjects ${ }^{(63)}$. Consent for data collection was obtained from participants during the DHS as per the DHS ethical approval protocol. DHS data use for this analysis was approved by the ICF, which is the implementing agency for the Demographic Health Surveys.

\section{Availability of data and material}

The datasets analysed in this study are publicly available at the DHS Program website ${ }^{(25)}$.

\section{Acknowledgements}

The authors would like to thank the DHS Program, ICF International for providing the data used in the analysis.

There was no funding directly for data analysis or writing this study. Funding for the DHS surveys is provided to the implementing agencies by the United States Agency for International Development.

P. K. M. conceptualised the idea and conducted a background check on the availability of the data. P. K. M. and F. H. designed the study analysis plan. T. N. M. conducted the literature search and synthesis and developed the study background. F. H. conducted the data analysis, results writing and contributing to the Methods section of the paper. P. K. M., F. H. and T. N. M. wrote the Discussion section, contributed to the intellectual development of the paper, attended to reviewers' comments, and finalised for submission. All authors read and approved the final manuscript.

P. K. M. is a capacity building advisor at the Global Programs for Research and Training, an affiliate of the University of California San Francisco. She is also faculty at Moi University, School of Public Health in Kenya. She holds a PhD in Nutritional Sciences (Stellenbosch University, South Africa), Master of Public Health (Moi University, Kenya) and Bachelor of Science in Food Nutrition and Dietetics (Egerton University, Kenya). P. K. M.'s public health research interest is in the nutrition transition, double burden of nutrition in low- and 
middle-income countries, maternal, infant and young child nutrition. She has interests in strengthening research capacity for health programmes and developing human capital for health systems in low- and middle-income countries.

F. H. is a statistician at the Global Programs for Research and Training, an affiliate of the University of California San Francisco. He holds a Master of Science in Epidemiology and Biostatistics, and a Bachelor of Science in Applied Statistics with Computing. F. H.'s research interest is on survey studies, clinical trials, cohort studies and data modelling on health-related events.

T. N. M. is a certified nutritionist working as a research officer in the Maternal, Infant and Young Child Nutrition Program within the Maternal and Child Wellbeing Unit at the African Population and Health Research Center. T. N. M. holds a BSc degree in Food, Nutrition and Dietetics from Kenyatta University and a graduate student of Master's degree in Food, Nutrition and Dietetics at Kenyatta University in Kenya. She has research interests in food and nutrition security, as well as maternal and child health issues.

The authors declare that they have no competing interests.

\section{Supplementary material}

The supplementary material for this article can be found at https://doi.org/10.1017/jns.2019.39

\section{References}

1. Tzioumis E \& Adair LS (2014) Childhood dual burden of underand overnutrition in low- and middle-income countries: a critical review. Food Nutr Bull 35, 230-243.

2. Doak CM, Adair LS, Bentley M, et al. (2005) The dual burden household and the nutrition transition paradox. Int J Obes (Lond) 29, 129-136.

3. Wibowo Y, Sutrisna B, Hardinsyah H, et al. (2015) Relationship between intra-household food distribution and coexistence of dual forms of malnutrition. Nutr Res Pract 9, 174-179.

4. Black RE, Victora CG, Walker SP, et al. (2013) Maternal and child undernutrition and overweight in low-income and middle-income countries. Lancet 382, 427-451.

5. Khan SH \& Talukder SH (2013) Nutrition transition in Bangladesh: is the country ready for this double burden. Obes Rev 14, Suppl. 2, 126-133.

6. Kimani-Murage E, Muthuri SK, Oti SO, et al. (2015) Evidence of a double burden of malnutrition in urban poor settings in Nairobi, Kenya. PLOS ONE 10, e0129943.

7. Wojcicki JM (2014) The double burden household in sub-Saharan Africa: maternal overweight and obesity and childhood undernutrition from the year 2000: results from World Health Organization Data (WHO) and Demographic Health Surveys (DHS). BMC Public Health 14, 1124.

8. Garrett JL \& Ruel MT (2003) Stunted Child-Overweight Mother Pairs: an Emerging Policy Concern? FCND Discussion Paper 148. http://ageconsearch.umn.edu/bitstream/16442/1/fc030148. pdf (accessed December 2019).

9. Masibo PK \& Makoka D (2012) Trends and determinants of undernutrition among young Kenyan children: Kenya Demographic and Health Survey; 1993, 1998, 2003 and 20082009. Public Health Nutr 15, 1715-1727.

10. Kenya National Bureau of Statistics (KNBS) and ICF International (2015) Kenya: Demographic and Health Survey
2014. http://dhsprogram.com/pubs/pdf/FR308/FR308.pdf (accessed December 2019).

11. World Health Organization (2010) Landscape Information System (NLIS) Country Profile: Indicators. Interpretation. http://www.who. int/nutrition/nlis_interpretation_guide.pdf (accessed December 2019).

12. Central Bureau of Statistics (Kenya), Ministry of Health (Kenya) \& ORC Macro (2004) Kenya Demographic and Health Survey, 2003. http://dhsprogram.com/pubs/pdf/FR151/FR151.pdf (accessed December 2019).

13. Kenya National Bureau of Statistics \& ICF Macro (2010) Kenya Demographic and Health Survey 2008-09. Calverton, MD: KNBS AND ICF Macro.

14. World Health Organization (2016) Strategic Action Plan to Reduce the Double Burden of Malnutrition in the South-East Asia Region 2016-2025. Colombo, Sri Lanka: WHO. https://apps.who.int/iris/bitstream/han dle $/ 10665 / 253377 /$ Strategic $\% 20$ Action $\% 20$ Plan $\% 20$ to $\% 20$ reduce $\%$ 20the $\% 20$ double $\% 20$ burden $\% 20$ of $\% 20$ malnutrition $\% 20$ in $\% 20$ SEAR \%202016-2025.pdf?sequence=1\&isAllowed $=\mathrm{y} \quad$ (accessed December 2019).

15. UNICEF (1990) Strategy for Improved Nutrition of Children and Women in Developing Countries. New York: UNICEF.

16. Victora CG, Adair L, Fall C, et al. (2008) Maternal and child undernutrition: consequences for adult health and human capital. Lancet 371, 340-357.

17. Swinburn BA, Sacks G, Hall KD, et al. (2011) The global obesity pandemic: shaped by global drivers and local environments. Lancet 378, 804-814.

18. Haddad L, Cameron L \& Barnett I (2015) The double burden of malnutrition in SE Asia and the Pacific: priorities, policies and politics. Health Policy Plan 30, 1193-1206.

19. Collares MF, Korevaar I. T, Hofman A, et al. (2017) Maternal thyroid function, prepregancy obesity and gestational weight gain The Generation R Study: a prospective cohort study. Clin Endocrinol (Oxf) 38, 42-49.

20. Louzada ML, Baraldi LG, Steele EM, et al. (2015) Consumption of ultra-processed foods and obesity in Brazilian adolescents and adults. Prev Med (Baltim) 81, 9-15.

21. Monteiro CA, Moubarac J-C, Levy RB, et al. (2018) Household availability of ultra-processed foods and obesity in nineteen European countries. Public Health Nutr 21, 18-26.

22. Kimani-Murage E \& Wekesah F (2015) Factors affecting actualisation of the WHO breastfeeding recommendations in urban poor settings in Kenya. Matern Child Nutr 11, 314-332.

23. Lee J, Houser RF, Must A, et al. (2010) Disentangling nutritional factors and household characteristics related to child stunting and maternal overweight in Guatemala. Econ Hum Biol 8, 188-196.

24. Barkin SL, Heerman WJ, Warren MD, et al. (2010) Millennials and the world of work :the impact of obesity on health and productivity. J Bus Psychol 25, 239-245.

25. Kenya National Bureau of Statistics (KNBS) and ICF International (2015) Kenya Demographic and Health Survey, 2014 (Dataset) KEIR72DT. https://dhsprogram.com/data/dataset/ Kenya_Standard-DHS_2014.cfm?flag=1 (accessed December 2019).

26. Demographic and Health Surveys (2008) Description of the demographic and health surveys individual recode data file. Calverton, MD: Measure DHS. https://www.dhsprogram.com/pubs/pdf/ DHSG4/Recode4DHS.pdf (accessed December 2019).

27. Croft TN, Marshall AMJ, Allen CK, et al. (2018) Guide to DHS Statistics. Rockville, MD: ICF. https://dhsprogram.com/Data/ Guide-to-DHS-Statistics/index.cfm (accessed December 2019).

28. Rutstein SO (2008) DHS Working Papers. The DHS Wealth Index: Approaches for Rural and Urban Areas, no. 60. https://www. dhsprogram.com/pubs/pdf/WP60/WP60.pdf (accessedDecember2019).

29. Rutstein S \& Johnson K (2004) The DHS Wealth Index. DHS Comparative Reports, no. 6. Calverton, MD: ORC Macro.

30. World Health Organization \& UNICEF Joint Monitoring Programme (JMP) for Water Supply and Sanitation (2016) Refining the definitions: an ongoing process and the ladder concept. 
https://www.wssinfo.org/definitions-methods/watsan-categories/ (accessed May 2017).

31. Masibo PK, Buluku E, Menya D, et al. (2013) Prevalence and determinants of under- and over-nutrition among adult Kenyan women; evidence from the Kenya Demographic and Health Survey 200809. East Afr J Public Health 10, 611-622.

32. Makoka D \& Masibo PK (2015) Is there a threshold level of maternal education sufficient to reduce child undernutrition? Evidence from Malawi, Tanzania and Zimbabwe. BMC Pediatr 15, 96.

33. Ezzati M, Lopez AD, Rodgers A, et al. (editors) (2004) Comparative Quantification of Health Risks: Global and Regional Burden of Disease Attributable to Selected Major Risk Factors, 1st ed. Geneva: WHO. http://apps.who.int/iris/bitstream/10665/42792/1/9241580348_ eng_Volume1.pdf (accessed December 2019).

34. Dieffenbach S \& Stein AD (2012) Stunted child/overweight mother pairs represent a statistical artifact, not a distinct entity. $J$ Nutr 142, 771-773

35. Ziraba AK, Fotso JC \& Ochako R (2009) Overweight and obesity in urban Africa: a problem of the rich or the poor? BMC Public Health 9, 465.

36. Gewa CA \& Yandell N (2012) Undernutrition among Kenyan children: contribution of child, maternal and household factors. Public Health Nutr 15, 1029-1038.

37. Shinsugi C, Gunasekara D, Gunawardena NK, et al. (2019) Double burden of maternal and child malnutrition and socioeconomic status in urban Sri Lanka. PLOS ONE 14, e0224222.

38. Boyle MH, Racine Y, Georgiades K, et al. (2006) The influence of economic development level, household wealth and maternal education on child health in the developing world. Soc Sci Med 63, 2242-2254.

39. Wamani H, Åstrøm AN, Peterson S, et al. (2007) Boys are more stunted than girls in sub-Saharan Africa: a meta-analysis of 16 demographic and health surveys. BMC Pediatr 7, 17.

40. Yang YY, Kaddu G, Ngendahimana D, et al. (2018) Trends and determinants of stunting among under-5s: evidence from the 1995, 2001, 2006 and 2011 Uganda Demographic and Health Surveys. Public Health Nutr 21, 2915-2928.

41. Condo JU, Gage A, Mock N, et al. (2015) Sex differences in nutritional status of HIV-exposed children in Rwanda: a longitudinal study. Trop Med Int Health 20, 17-23.

42. Lawrence RA \& Lawrence RM (2011) Breastfeeding: A Guide for The Medical Profession, 7th ed. Maryland Heights, MI: Elsevier Mosby.

43. Shetty P (2005) Female nutritional status across the life-span in sub-Saharan Africa 1. Prevalence patterns. Public Health Nutr 8, 929-931.

44. Kramer MS \& Kakuma R (2004) The optimal duration of exclusive breastfeeding: a systematic review. Adv Exp Med Biol 554, 63-77.

45. Onyango AW, Esrey SA \& Kramer MS (1999) Continued breastfeeding and child growth in the second year of life: a prospective cohort study in western Kenya. Lancet 354, 2041-2045.

46. Owais A, Kleinbaum D, Suchdev P, et al. (2014) Household food security and infant feeding practices in rural Bangladesh. Public Health Nutr 19, 1875-1881.
47. Lutter CK, De Onis M, Monica T, et al. (2011) Undernutrition, poor feeding practices, and low coverage of key nutrition interventions. Pediatrics 128, e1418-e1427.

48. Amugsi DA, Mittelmark MB, Lartey A, et al. (2014) Influence of childcare practices on nutritional status of Ghanaian children: a regression analysis of the Ghana demographic and health surveys. BMJ Open 4, e005340.

49. Ersino G, Henry CJ \& Zello GA (2016) Suboptimal feeding practices and high levels of undernutrition among infants and young children in the rural communities of Halaba and Zeway, Ethiopia. Food Nutr Bull 37, 409-424.

50. Gautam KP, Adhikari M, Khatri RB, et al. (2016) Determinants of infant and young child feeding determinants of infant and young child feeding practices in Rupandehi, Nepal. BMC Res Notes $9,135$.

51. Jehn M \& Brewis A (2009) Paradoxical malnutrition in motherchild pairs: untangling the phenomenon of over- and undernutrition in underdeveloped economies. Econ Hum Biol 7, 28-35.

52. Popkin BM, Adair LS \& Ng SW (2013) Global nutrition transition and the pandemic of obesity in developing countries. Nutr Rev 70, 3-21.

53. Bezerra IN, Curioni C \& Sichieri R (2018) Association between eating out of home and body weight. Nutr Rev 70, 65-79.

54. Lakati A, Binns C \& Stevenson MR (2002) Breast-feeding and the working mother in Nairobi. Public Health Nutr 5, 715-718.

55. Al-matary A \& Ali J (2013) The impact of child-rearing by maids on mother-child attachment. Hamdan Med J 6, 197-204.

56. Gielen AC, Faden RR, O'Campo P, et al. (1991) Maternal employment during the early postpartum period: effects on initiation and continuation of breast-feeding. Pediatrics 87, 298-305.

57. Chuang C, Chang P, Chen Y, et al. (2010) Maternal return to work and breastfeeding: a population-based cohort study. Int J Nurs Stud 47, 461-474.

58. Chuang C-H, Chang P-J, Hsieh W-S, et al. (2007) The combined effect of employment status and transcultural marriage on breast feeding: a population-based survey in Taiwan. Paediatr Perinat Epidemiol 21, 319-329.

59. Hawkins SS, Griffiths LJ, Dezateux C, et al. (2007) Maternal employment and breast-feeding initiation: findings from the Millennium Cohort Study. Paediatr Perinat Epidemiol 21, 242-247.

60. Tsai S-Y (2013) Impact of a breastfeeding-friendly workplace on an employed mother's intention to continue breastfeeding after returning to work. Breastfeed Med 8, 210-216.

61. Keding G (2016) Nutrition transition in rural Tanzania and Kenya. World Rev Nutr Diet 115, 68-81.

62. Bassete MN, Romaguera D, Giménez MA, et al. (2014) Prevalence and determinants of the dual burden of malnutrition at the household level in Puna and Quebreda of Humahuaca, Jujuy, Argentina. Nutr Hosp 29, 322-330.

63. ICF International (2017) The DHS Program: Protecting the Privacy of DHS Survey Respondents. https://dhsprogram.com/What-We-Do/ Protecting-the-Privacy-of-DHS-Survey-Respondents.cfm (accessed September 2017). 